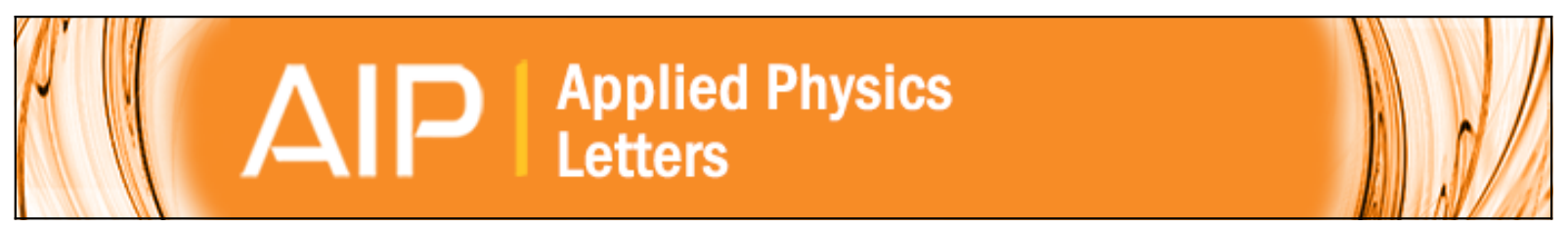

\title{
Chemical environment of rare earth ions in Ge28.125Ga6.25S65.625 glass-ceramics
} doped with Dy3+

Rongping Wang, Kunlun Yan, Mingjie Zhang, Xiang Shen, Shixun Dai, Xinyu Yang, Zhiyong Yang, Anping Yang

, Bin Zhang, and Barry Luther-Davies

Citation: Applied Physics Letters 107, 161901 (2015); doi: 10.1063/1.4934261

View online: http://dx.doi.org/10.1063/1.4934261

View Table of Contents: http://scitation.aip.org/content/aip/journal/apl/107/16?ver=pdfcov

Published by the AIP Publishing

\section{Articles you may be interested in}

Conductivity study on GeS2-Ga2S3-Agl-Ag chalcohalide glasses

J. Appl. Phys. 114, 023701 (2013); 10.1063/1.4813139

Upconversion luminescence in Er3+ doped Ga10Ge25S65 glass and glass-ceramic excited in the near-infrared J. Appl. Phys. 113, 083520 (2013); 10.1063/1.4793638

Mechanism of the enhancement of mid-infrared emission from GeS2-Ga2S3 chalcogenide glass-ceramics doped with $\mathrm{Tm} 3+$

Appl. Phys. Lett. 100, 231910 (2012); 10.1063/1.4727900

Optical spectroscopy and upconversion luminescence in Nd 3 + doped Ga 10 Ge 25 S 65 glass

J. Appl. Phys. 106, 103512 (2009); 10.1063/1.3259439

Photostimulated luminescence in a rare earth-doped fluorobromozirconate glass ceramic

Appl. Phys. Lett. 75, 2386 (1999); 10.1063/1.125022

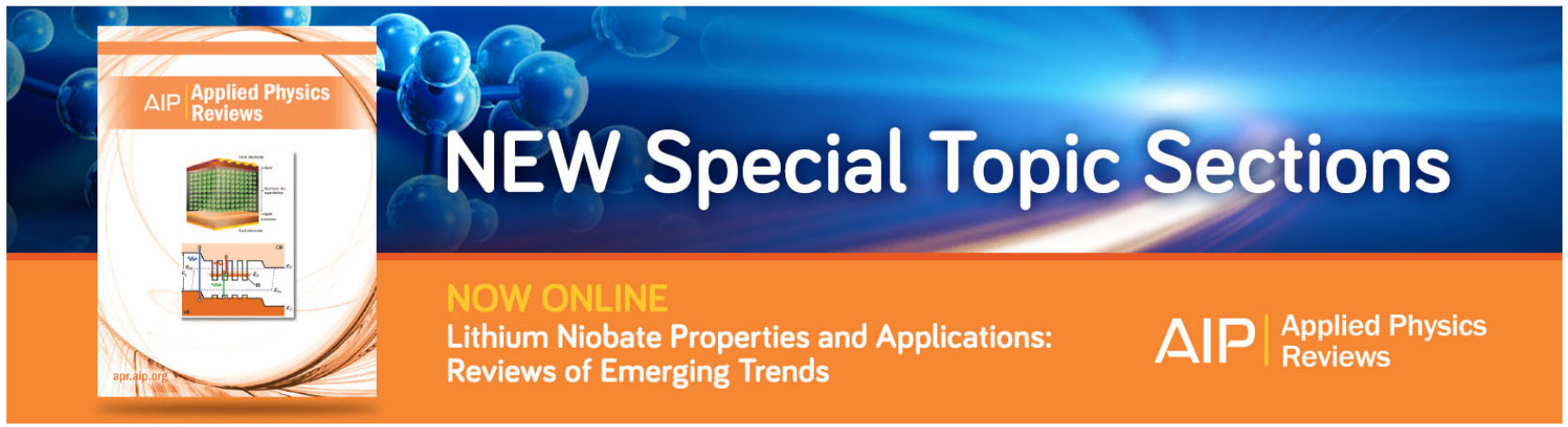




\title{
Chemical environment of rare earth ions in $\mathrm{Ge}_{28.125} \mathrm{Ga}_{6.25} \mathrm{~S}_{65.625}$ glass-ceramics doped with $\mathrm{Dy}^{3+}$
}

\author{
Rongping Wang, ${ }^{1, a)}$ Kunlun Yan, ${ }^{1}$ Mingjie Zhang, ${ }^{2}$ Xiang Shen, ${ }^{3}$ Shixun Dai, ${ }^{3}$ Xinyu Yang, ${ }^{4}$ \\ Zhiyong Yang, ${ }^{1,2}$ Anping Yang, ${ }^{2}$ Bin Zhang, ${ }^{2}$ and Barry Luther-Davies ${ }^{1}$ \\ ${ }^{1}$ Centre for Ultrahigh bandwidth Devices for Optical Systems, Laser Physics Centre, Research School of \\ Physics and Engineering, The Australian National University, Canberra ACT 2600, Australia \\ ${ }^{2}$ Jiangsu Key Laboratory of Advanced Laser Materials and Devices, School of Physics and Electronic \\ Engineering, Jiangsu Normal University, Xuzhou, Jiangsu 221116, China \\ ${ }^{3}$ Laboratory of Infrared Material and Devices, The Advanced Technology Research Institute, \\ Ningbo University, Ningbo 315211, China \\ ${ }^{4}$ Faculty of Chemistry and Material Engineering, Wenzhou University, Wenzhou 325027, China
}

(Received 14 September 2015; accepted 9 October 2015; published online 19 October 2015)

We have annealed $\mathrm{Ge}_{28.125} \mathrm{Ga}_{6.25} \mathrm{~S}_{65.625}$ glasses doped with $0.5 \%$ Dy to create glass-ceramics in order to examine the local chemical environment of the rare earth ions (REI). More than 12 times enhancement of the emission at 2.9 and $3.5 \mu \mathrm{m}$ was achieved in glass-ceramics produced using prolonged annealing time. Elemental mapping showed clear evidence that $\mathrm{Ga}_{2} \mathrm{~S}_{3}$ crystalline grains with a size of $50 \mathrm{~nm}$ were dispersed in a Ge-S glass matrix in the glass-ceramics, and the REI could only be found near the $\mathrm{Ga}_{2} \mathrm{~S}_{3}$ crystalline grains. From the unchanged lineshape of the emissions at 2.9 and $3.5 \mu \mathrm{m}$ and lack of splitting of the absorption peaks, we concluded that the REI were bonded to $\mathrm{Ga}$ on the surface of the $\mathrm{Ga}_{2} \mathrm{~S}_{3}$ crystals. (C) 2015 AIP Publishing LLC.

[http://dx.doi.org/10.1063/1.4934261]

Chalcogenide glasses have high linear and nonlinear refractive indices, a wide transparency window (up to $25 \mu \mathrm{m}$ ) and low phonon energy and, thus, are considered one of the best materials to host rare earth ions (REI) for the potential applications as lasers, amplifiers, and high-brightness infrared sources for remote sensing. ${ }^{1-5}$ While the mid-infrared emissions from transitions between different energy levels are easily quenched in silica and fluoride glasses because of strong multi-phonon relaxation induced by the high phonon energy in silica $\left(1100 \mathrm{~cm}^{-1}\right)$ and fluoride glasses $\left(560 \mathrm{~cm}^{-1}\right)$, it is expected that active emissions in REI doped chalcogenide glasses could be achieved due to their comparatively low phonon energy $\left(150-450 \mathrm{~cm}^{-1}\right) .{ }^{1,2,6}$ In fact, the emissions due to the transitions between the different energy levels of REI have been observed, ${ }^{7,8}$ and modelling of laser action in REI-doped chalcogenide glasses has indicated that it should be possible to achieve lasing in the wavelength range from 3 to $5 \mu \mathrm{m}$. However, only one successful experiment on lasing has been reported using REI doped chalcogenide glasses, ${ }^{10}$ and the lasing wavelength still lay in the near infrared.

Recently, it has been found that tuning the chemical environment of the REI through crystallization is an efficient way to enhance the luminescence efficiency. ${ }^{11,12}$ For example, enhanced fluorescence was obtained in low-loss oxide glass-ceramic waveguides, making them quite promising for integrated optoelectronic devices. ${ }^{13}$ Similar behaviour was also observed in REI-doped chalcogenide glass-ceramics where the luminescence increased significantly in transparent glass-ceramics compared with that of the base glass. ${ }^{11,12,14}$ However, the fundamental mechanism for the enhancement of the mid-IR emission upon crystallization and the local

\footnotetext{
${ }^{\text {a) }}$ Author to whom correspondence should be addressed. Electronic mail: rongping.wang@anu.edu.au
}

chemical environment of REI-ion still remain largely unknown.

In this paper, we concentrated on fundamental issues when creating controllable and reproducible REI-doped chalcogenide glass-ceramics, such as the formation of different crystalline phases and the chemical environment of REI within them. The emissions at 2.9 and $3.5 \mu \mathrm{m}$ were found to increase significantly with prolonged annealing time. Combined elemental mapping with other optical techniques, it was found that $\mathrm{Ga}_{2} \mathrm{~S}_{3}$ crystals with a size of around $50 \mathrm{~nm}$ were homogeneously dispersed in the glass matrix, and the REI were bonded with $\mathrm{Ga}_{2} \mathrm{~S}_{3}$ on the surface of those crystals.

Chemically stoichiometric $\mathrm{Ge}_{28.125} \mathrm{Ga}_{6.25} \mathrm{~S}_{65.625}$ glass doped with $0.5 \mathrm{wt} \%$ Dy was synthesized by the meltquenching method. The glass rod was annealed and cut into glass disks $(\varnothing 10 \mathrm{~mm} \times 1.0 \mathrm{~mm})$, and finally polished to optical quality on both sides for optical measurements. The glass transition temperature, $\mathrm{T}_{\mathrm{g}}$, of the as-prepared glasses was measured using a differential scanning calorimeter (DSC1, Mettler-Toledo, Ltd., Switzerland). The glass-ceramics were created by annealing the as-prepared glass under nitrogen gas at $415^{\circ} \mathrm{C}$, which is $20^{\circ} \mathrm{C}$ higher than $\mathrm{T}_{\mathrm{g}}$. The chemical compositions of the glasses were analysed by an energy dispersive $x$-ray spectrometer (EDS) installed on a scanning electron microscope using commercial $\mathrm{Ge}_{33} \mathrm{As}_{12} \mathrm{Se}_{55}$ as a reference.

The mid-IR fluorescence spectra in the range of 2000-4100 $\mathrm{nm}$ were measured in reflection mode through a computer-controlled system consisting of a Zolix Omnik3015 monochromator with a SCITEC Model 420 lock-in amplifier and an InSb detector cooled with liquid nitrogen. A laser at $1300 \mathrm{~nm}$ was used as an excitation beam. All measurements were taken at room temperature. 


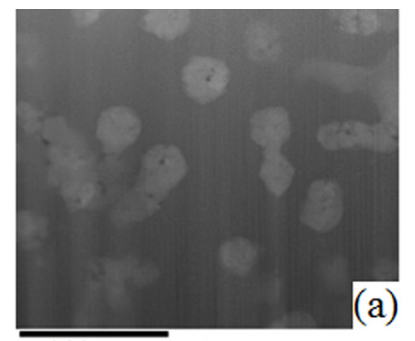

$200 \mathrm{~nm}$
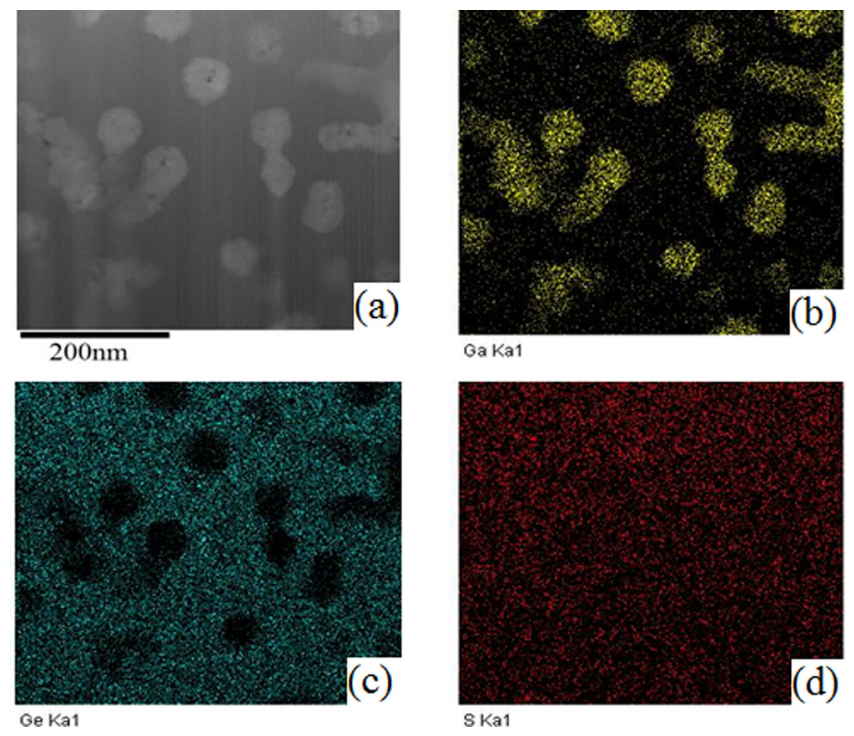

FIG. 1. (a) Typical morphology of glass-ceramics: (b) Ga-, (c) Ge-, and (d) S-element mapping in the same area as (a).

The microstructure of the glass-ceramics was examined by transmission electron microscopy (TEM, FEI Tecnai TF20) operated at $200 \mathrm{kV}$. The elemental distributions in glassceramics were analysed using Oxford INCA EDS equipped in TEM with a spatial resolution of $5 \mathrm{~nm}$.

We first examined the chemical compositions using EDS installed on a scanning electron microscope since it was important to ensure that thermal annealing did not alter the chemical compositions of the samples. The experimental data were averaged from 10 different positions of the samples, and the spot size of the electron beam is around $3 \mu \mathrm{m}$. The as-prepared glass with a composition of $\mathrm{Ge}_{28.16} \mathrm{Ga}_{6.65} \mathrm{~S}_{65.09}$ and glass-ceramics annealed at $415^{\circ} \mathrm{C}$ for $50 \mathrm{~h}$ with a composition of $\mathrm{Ge}_{28.73} \mathrm{Ga}_{6.76} \mathrm{~S}_{64.49}$ are very close to the nominal composition of the glass $\mathrm{Ge}_{28.125} \mathrm{Ga}_{6.25} \mathrm{~S}_{65.325}$. Moreover, we did not observe any significant trace of the oxygen in the glass-ceramics due to the protection of the nitrogen gas during the ceramization process. Instead, we have found that, while the glasses with an annealing time less than $15 \mathrm{~h}$ show no obvious changes (judged by eye) in the surface, once the annealing time is more than $15 \mathrm{~h}$, the transparency of the glass-ceramics decreases due to the formation of the crystalline particles in the samples.

We employed TEM to characterize the microstructure and elemental distributions in the glass-ceramics. Figure 1(a) shows the typical morphology of the glass-ceramics, from which well-dispersed white and round particles with a size of around $50 \mathrm{~nm}$ are evident. We detected the elemental distributions in the same area using EDS mapping which would be better suited to this sample compared with electron energy loss spectra (EELS) as used in similar investigations, ${ }^{14}$ because the Dy M-edge lies just beyond the Ge L-edge and Ga L-edge and would be impacted by their background tails, especially when Dy was expected to be present only in very small amounts. In contrast, in EDS, the Dy L-alpha peak at $6.5 \mathrm{keV}$ does not show any overlap with the other elements.

Figures 1(b)-1(d) are, respectively, Ga, Ge, and S elemental mapping from the same area as shown in Fig. 1(a).

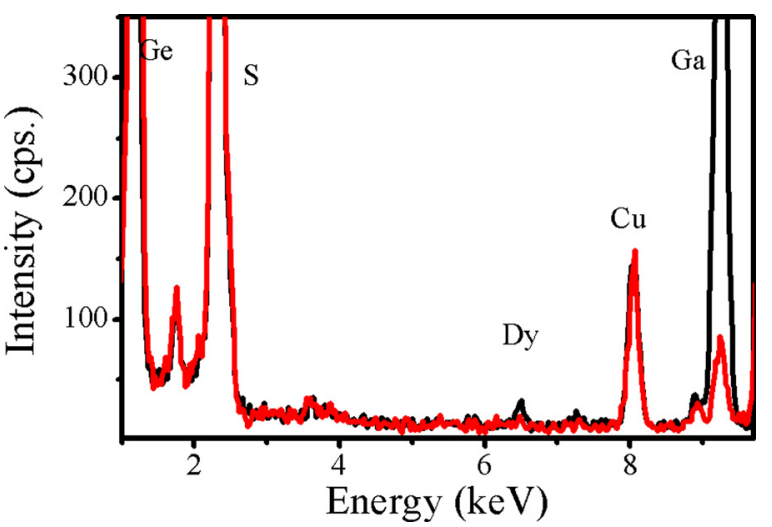

FIG. 2. EDS spectra in amorphous (red) and crystalline (black) parts of Fig. 1 (a), where the weak peak at $6.5 \mathrm{keV}$ is evidence of the Dy distribution in Ga-rich area.

Apparently, Figs. 1(a)-1(c) show similar morphology, and thus, the yellow dots in Fig. 1(b) indicate that Ga-element is dominant in the white crystalline grains in Fig. 1(a), while the uniform blue color in Fig. 1(c) indicates that Ge is uniformly distributed in the amorphous parts of the glassceramic. In contrast, Sulphur-elemental mapping across the whole area exhibit uniform dark-red, indicating that $\mathrm{S}$ is homogeneously dispersed over the entire region.

Regarding the distribution of Dy, the EDS signal is rather weak because the nominal content of $0.5 \%$ Dy is close to the resolution limit of EDS. However, we measured more than 18 points located in either the amorphous or crystalline parts over longer exposure times in order to get the reproducible and reliable results on the distribution of Dy. It was found that Dy usually bonded with Ga-related structure while it was hard to find any trace of Dy in the amorphous parts. Two curves in Fig. 2 showed the typical EDS results with long exposure times from the $\mathrm{Ga}-\mathrm{S}$ and $\mathrm{Ge}-\mathrm{S}$ regions, respectively. As we can see, trace amounts of the Dy could be detected in the Ga-S grains whilst nothing could be found in the amorphous $\mathrm{Ge}-\mathrm{S}$ parts, and the signal of the $\mathrm{Cu}$ is from $\mathrm{Cu}$ grid of TEM sample holder.

Figure 3(a) shows the mid-infrared emission from the glass and glass-ceramics excited by a $1300 \mathrm{~nm}$ laser, where a significant enhancement of the emission in the glassceramics with prolonged annealing time is evident. For example, emission at $2.9 \mu \mathrm{m}$ due to the Dy ${ }^{6} \mathrm{H}_{13 / 2} \rightarrow{ }^{6} \mathrm{H}_{15 / 2}$ transition is enhanced more than 12 times, which is in sharp contrast with fivefold increase in $\mathrm{Tm}^{3+}$ doped chalcogenide glass-ceramics. ${ }^{14}$ The emission at $3.5 \mu \mathrm{m}$, which has been observed in $\mathrm{Dy}^{3+}$-doped $\mathrm{BaYb}_{2} \mathrm{~F}_{8}$ and $\mathrm{Ga}-\mathrm{Sb}-\mathrm{S}$ glasses, ${ }^{15,16}$ also appears and its intensity increases 8 times upon thermal annealing. However, the shapes of the peaks do not change, indicating that REI ions are not located in the crystalline structure of the glass-ceramics otherwise significant narrowing of the emission peaks should be observed. In addition, we only observed large absorption in glass-ceramics compared with that in pure glass, but we did not observe any splitting of the REI absorption peaks as shown in Fig. 3(b). It is well known that crystal field effects could induce the splitting of the absorption peaks if the REI ions are located within the crystallites. Both the unchanged lineshape of the emission peaks in Fig. 3 and lack of splitting of the 

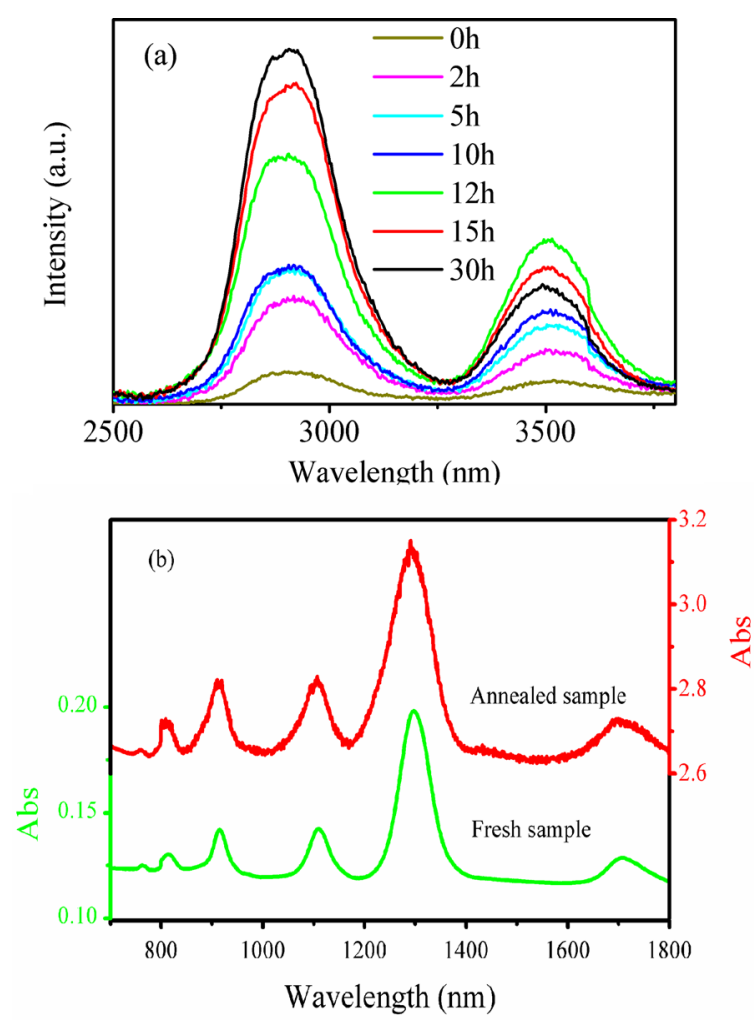

FIG. 3. (a) Photoluminescence of the glass and glass-ceramics excited by $1300 \mathrm{~nm}$ laser, and (b) absorption spectra of the fresh glass and glassceramics annealed at $415^{\circ} \mathrm{C}$ for $30 \mathrm{~h}$.

absorption peaks in Fig. 3(b) confirm that the REI ions are not within the Ga-S crystals.

In the past decade, several different approaches have been used to identify the chemical environment of REI ions in chalcogenide glasses and glass-ceramics. It has been widely accepted that the addition of Ga into a chalcogenide glass can improve the solubility of REI ions in the matrix. ${ }^{6}$ Lee et al. employed site-selective excitation spectra and found preferential spatial correlations between $\mathrm{Nd}^{3+}$ and $\mathrm{GaS}_{4}$ tetrahedra even at low Ga-doping levels. ${ }^{17}$ Using $a b$ initio molecular dynamics simulations, they further confirmed that Ga-Nd bonds were formed preferentially. On the other hand, in a recent paper, Lin et al. proposed that $\mathrm{Tm}^{3+}$ ions were not involved in the nucleation and growth of $\mathrm{Ga}_{2} \mathrm{~S}_{3}$ crystals from almost identical XRD patterns of glassceramics with and without REI doping. ${ }^{14}$ However, in both our experiments and those of Ref. 14, crystal grains with a size around $50 \mathrm{~nm}$ are observed in the glass-ceramics, and such a small size can induce broad XRD and Raman peaks. Moreover, the content of REI doping is less than $0.5 \%$. Compared with broad peaks induced by the size effect, slightly structural modification by low level doping is too small to be observed. Therefore, the evidence of the chemical environment of REI-ions related to $\mathrm{Ga}_{2} \mathrm{~S}_{3}$ crystalline grains from XRD patterns is arguable. In contrast, our EDS mapping in Figs. 1(a)-1(d) shows clear proof that REI-ions are not registered at the amorphous Ge-S matrix.

The result from Figs. 1 and 2 is in contradiction with that in Ref. 14, where Lin et al. reported that the REI ions were dispersed at the Ge-rich sites in the glass matrix. We note that extraction of the Ga-L3 edge from the broad background from the Ge- $\mathrm{L}_{3}$ edge in EELS in the paper by Lin et al. could be a major problem since their $\mathrm{L}_{3}$ edges are close to each other, and therefore, it is hard to distinguish Ge- and Ga-related signals. In contrast, our selective elemental mapping in Fig. 1 clearly shows that Ge- and Ga-rich regions are distinguishable, and $\mathrm{Ga}_{2} \mathrm{~S}_{3}$ crystals are uniformly dispersed in an amorphous Ge-S network. While the experimental evidence confirms that the REI ions are not registered within both $\mathrm{Ga}_{2} \mathrm{~S}_{3}$ grains and Ge-S glass matrix, a reasonable explanation from our results is that, upon crystallization, Dy ions migrate/segregate to the interface between nanocrystalline $\mathrm{Ga}_{2} \mathrm{~S}_{3}$ and surrounding glass matrix, and the REI ions are actually bonded to the $\mathrm{Ga}_{2} \mathrm{~S}_{3}$ crystals forming Ga-REI or Ga-S-REI bonds at that surface (or interface between crystalline and amorphous parts in glass-ceramics). The central result regarding the local chemical environment of REI is in excellent agreement with that from the site-selective excitation spectra in which Ga-REI bonds were confirmed to be formed preferentially. ${ }^{17}$

We further explained why we can observe the trace of $\mathrm{Dy}^{3+}$ in Fig. 2 related to $\mathrm{Ga}_{2} \mathrm{~S}_{3}$ grains. While detecting the chemical composition, although the beam was focused on the surface of the white crystals in Fig. 1(a), the EDS information from the black curve in Fig. 2 can contain contributions from both the surface and interior of the nano-crystals as well as adjacent amorphous regions due to the possible shift of the focused beam during the prolonged exposure time. If we reduced the size of the focused beam to $5 \mathrm{~nm}$ and then reduced the possible shift of the focused beam, there is a contribution from the REI ions bonded with Ga on the top of the white crystals. On the other hand, while the beam was focused on the amorphous regions with large area, any shift in the position of the beam means that it still remains within the amorphous zone. Therefore, the shift of the beam has no effect on the signal of the compositions in the regions. Most importantly, if the Ga-REI or Ga-S-REI bonds exist on the surface of the crystallites rather than within them, one would not expect to observe narrowing of the emission peak or splitting of the absorption bands due to crystalline field effects, and this is in accord with the experimental observations.

Finally, we analysed the mechanism of the photoluminescence enhancement in the chalcogenide glass-ceramics. In the oxide glass-ceramics, the low phonon-energy crystalline environment formed by the incorporation of REI-ions into the crystalline lattice during the ceramization process is considered the key factor for the luminescence enhancement. ${ }^{18}$ Obviously, in our case, the evidence on the position of the REI ions in Fig. 1 rules out the possibility that the REI ions are incorporated into the $\mathrm{Ga}_{2} \mathrm{~S}_{3}$ lattice. Lin et al. proposed that the formation of Ge-rich areas, rather than the presence of crystallites in the glass-ceramics is more favourable to the mid-infrared photoluminencence. ${ }^{14}$ If this is a case, stronger photoluminescence should be observed in the Ge-rich glasses. We prepared two glasses with different Ge composition (one is chemically stoichiometric $\mathrm{Ge}_{26.67} \mathrm{Ga}_{8} \mathrm{~S}_{65.33}$ and the other is Ge-rich $\mathrm{Ge}_{29.6} \mathrm{Ga}_{8} \mathrm{~S}_{62.4}$ glass, both doped with $0.5 \%$ Dy) and measured their photoluminescence. However, we did not observe any correlation between the intensity of the photoluminescence and the Ge content. We believe that an increase of the refractive index 
of the host matrix will lead to increased spontaneous emission probabilities and thus enhanced photoluminescence. ${ }^{19,20}$ This especially happens when $\mathrm{Ga}_{2} \mathrm{~S}_{3}$ crystals are induced in glass-ceramics since $\mathrm{Ga}_{2} \mathrm{~S}_{3}$ has a large refractive index of $2.563,{ }^{21}$ which is in contrast with the host glass with a refractive index of 2.122 at $1.55 \mu \mathrm{m}$. Significant role of $\mathrm{Ga}$ in enhancing the photoluminescence of the glass-ceramics is thus two-folded: one is to increase the refractive index of the glass-ceramics via the formation of the $\mathrm{Ga}_{2} \mathrm{~S}_{3}$ crystals, and another is to prevent the formation of the REI-ion clusters that could quench the photoluminescence.

In summary, we have prepared $\mathrm{Ge}_{28.125} \mathrm{Ga}_{6.25} \mathrm{~S}_{65.625}$ glasses doped with $0.5 \%$ Dy and annealed the glasses with different durations in order to probe the local chemical environment of REI ions in the chalcogenide glass-ceramics. Emission at 2.9 and $3.5 \mu \mathrm{m}$ was found to be more than 12 times enhanced after prolonged annealing times. Elemental mapping showed clear evidence that in the interior of the glass-ceramics, $\mathrm{Ga}_{2} \mathrm{~S}_{3}$ crystalline grains with a size of $50 \mathrm{~nm}$ were dispersed in the Ge-S glass matrix, and REI ions only can be found in the vicinity of the $\mathrm{Ga}_{2} \mathrm{~S}_{3}$ nanocrystals. From the unchanged lineshape of the emission peaks and lack of splitting of the absorption peaks, we concluded that REI ions are bonded with $\mathrm{Ga}$ on the surface of the $\mathrm{Ga}_{2} \mathrm{~S}_{3}$ crystals.

This research was partly supported by the Australian Research Council through its Centres of Excellence Grant CE110001018, Discovery Project Grant DP110102753 and Discovery Early Career Researcher Award Grant DE 120101036.

${ }^{1}$ K. Tanaka and K. Shimakawa, Amorphous Chalcogenide Semiconductors and Related Materials (Springer, New York, 2011).
${ }^{2}$ R. P. Wang, Amorphous Chalcogenides: Advances and Applications (Pan Stanford Publishing, Singapore, 2014).

${ }^{3}$ B. J. Eggleton, B. Luther-Davies, and K. Richardson, Nat. Photonics 5, 141-148 (2011).

${ }^{4}$ S. D. Jackson, Nat. Photonics 6, 423-431 (2012).

${ }^{5}$ Y. Yu, X. Gai, P. Ma, D. Y. Choi, Z. Yang, R. Wang, S. Debbarma, S. J. Madden, and B. Luther-Davies, Laser Photonics Rev. 8, 792-798 (2014).

${ }^{6}$ A. B. Seddon, Z. Tang, D. Furniss, S. Sujecki, and T. M. Benson, Opt. Express 18, 26704-26719 (2010).

${ }^{7}$ A. A. Mak and B. M. Antipenko, J. Appl. Spectrosc. 37, 1458 (1982).

${ }^{8}$ J. S. Sanghera, L. Brandon Shaw, and I. D. Aggarwal, IEEE J. Sel. Top. Quantum Electron. 15(1), 114-119 (2009).

${ }^{9}$ R. S. Quimby, L. B. Shaw, J. S. Sanghera, and I. D. Aggarwal, IEEE Photonics Technol. Lett. 20(2), 123-125 (2008).

${ }^{10}$ T. Schweizer, D. W. Hewak, D. M. Payne, T. Jensen, and G. Huber, Electron. Lett. 32, 666-667 (1996).

${ }^{11}$ R. Balda, S. García-Revilla, J. Fernández, V. Seznec, V. Nazabal, X. H. Zhang, J. L. Adam, M. Allix, and G. Matzen, Opt. Mater. 31(5), 760-764 (2009).

${ }^{12}$ E. Guillevic, M. Allix, X. Zhang, J.-L. Adam, G. Matzen, and X. Fan, Mater. Res. Bull. 45(4), 448-455 (2010).

${ }^{13}$ S. N. B. Bhaktha, F. Beclin, M. Bouazaoui, B. Capoen, A. Chiasera, M. Ferrari, C. Kinowski, G. C. Righini, O. Robbe, and C. Turrell, Appl. Phys. Lett. 93, 211904 (2008).

${ }^{14}$ C. Lin, S. Dai, C. Liu, B. Song, Y. Xu, F. Chen, and J. Heo, Appl. Phys. Lett. 100, 231910 (2012).

${ }^{15}$ N. Djeu, V. E. Hartwell, A. A. Kaminskii, and A. Butashin, Opt. Lett. 22, 997-999 (1997).

${ }^{16}$ M. Zhang, A. Yang, Y. Peng, B. Zhang, H. Ren, W. Guo, Y. Yang, C. Zhai, Y. Wang, Z. Yang, and D. Tang, Mater. Res. Bull. 70, 55-59 (2015).

${ }^{17}$ T. H. Lee, S. I. Simdyankin, J. Hegedus, J. Heo, and S. R. Elliott, Phys. Rev. B 81, 104204 (2010).

${ }^{18}$ D. Chen, Y. Yu, P. Huang, F. Weng, H. Lin, and Y. Wang, Appl. Phys. Lett. 94, 041909 (2009).

${ }^{19}$ M. Hubert, L. Calvez, X. Zhang, and P. Lucas, Opt. Mater. 35, 2527 (2013).

${ }^{20}$ Yu. S. Tver'yanovich and A. Tverjanovich, in Semiconducting Chalcogenide Glass III, edited by R. Fairman and B. Ushkov (Elsevier, Academic Press, Amsterdam, 2004), pp. 169-207.

${ }^{21}$ M. Zhang, X. Jiang, L. Zhou, and G. Guo, J. Mater. Chem. C 1, 4754 (2013). 